\title{
MONTE-CARLO CALCULATIONS FOR NEUTRON YIELD FROM PHOTONUCLEAR REACTIONS FOLLOWING BREMSSTRAHLUNG IN TUNGSTEN TARGET
}

\author{
N. T. Khai, T. D. Thiep, T. T. An, P. V. Cuong, N. T. Vinh \\ Institute of Physics, Hanoi, Vietnam
Yu. P. Gangrski, G. V. Mishinski, V.I. Zhemenik, Sh. Gerbish, A. G. Belov, O.D. Maslov

Joint Institute for Nuclear Research, Dubna

\begin{abstract}
The photonuclear reactions of $(\gamma, x n)$ or $(\gamma, x n p)$ types can be used to produce high-intensity neutron sources for research and applied purposes. In this work a Monte-Carlo calculation has been used to evaluate the production yields of neutrons from the $(\gamma, n)$ and $(\gamma, 2 n)$ reactions following the bremsstrahlung produced by 100 and $200 \mathrm{MeV}$ electron beams on the tungsten target. Our calculations indicated that the neutron yield is an increasing function of the target thickness in a considered range from 1.5 to $2.5 \mathrm{~mm}$.

Фотоядерные реакции $(\gamma, x n)$ или $(\gamma, x n p)$ могут быть использованы в производстве высокоинтенсивных нейтронных источников для исследовательских и прикладных целей. В этой работе был использован метод Монте-Карло для оценки выхода нейтронов в реакциях $(\gamma, n)$ и $(\gamma, 2 n)$ с тормозным излучением, произведенным облучением вольфрамовой мишени электронами с энергией 100 и 200 МэВ. Наши расчеты показали, что выход нейтронов увеличивается с увеличением рассмотренных толщин мишеней от 1,5 до 2,5 мм.
\end{abstract}

PACS: 25.20.-X

\section{INTRODUCTION}

The bremsstrahlung emissions produced by accelerated electron beams are intense and high-energy photon sources. They are widely used in photonuclear reaction research and applied nuclear physics. In electron accelerators, tungsten (W) is often used as a target because it has a large cross section for bremsstrahlung production, a high melting temperature and good heat conductivity [1]. Moreover, for the tungsten isotopes, ${ }^{180,182-184,186} \mathrm{~W}$, the cross sections of the $(\gamma, n)$ and $(\gamma, 2 n)$ reactions are relatively high [2]. Therefore, these reactions can be used to produce secondary neutrons for research purposes during accelerator operation [3]. The electron accelerators which can be used to produce secondary neutrons using different electron energies are Microtron MT-25 at the Laboratory of Nuclear Reactions and LUE-200 at the Laboratory of Neutron Physics, Joint Institute for Nuclear Research, 
Dubna, Russia and Linear Electron Accelerator at Pohang Accelerator Center, Korea. In this paper we show that the $(\gamma, n)$ and $(\gamma, 2 n)$ reactions mentioned above provide a high-intensity neutron source by evaluating the expected neutron yield in the cases of using the 100 and $200 \mathrm{MeV}$ electron beams incident on the thick tungsten target. In the first step the energy and angular distributions of the bremsstrahlung photons are evaluated, using both theoretical models and experimental data, especially those related to non-zero emission angles [4-6]. In the second step a folding with the reaction cross sections gives an estimation of the production yield of neutrons from the photonuclear reactions of interest. The consideration has been carried out at three values $1.5,2.0$ and $2.5 \mathrm{~mm}$ of the target thickness for both cases of the 100 and $200 \mathrm{MeV}$ incident electron energies.

\section{TOTAL NEUTRON YIELD}

Bremsstrahlung photons can be emitted whenever a charged particle experiences a change in momentum under the influence of the Coulomb field of a nucleus. The rate of energy loss due to bremsstrahlung and the cross section for its production are inversely proportional to the square of the mass of the incident particle [7]:

$$
\begin{gathered}
d E_{b} / d t \sim Z^{2} Z_{t}^{2} / m^{2}, \\
\sigma_{b} \sim Z_{t}^{2}\left(e^{2} / m c^{2}\right)^{2},
\end{gathered}
$$

where $m$ and $Z$ are, respectively, the mass and the charge of the beam particle, and $Z_{t}$ is the atomic number of the target. Bremsstrahlung emission is, therefore, a major energy loss mechanism for electrons, the lightest charged particle, especially at relativistic energies greater than a few $\mathrm{MeV}$.

At very low electron energies the angular distribution of bremsstrahlung is maximum in a direction perpendicular to the incident beam [7,8]. However, as the energy is increased, the maximum occurs at increasingly forward angles and in the limit of very high energies, the emission of bremsstrahlung essentially occurs in a narrow cone in the forward direction. The root-mean-square (rms) angle of emission is then given by [7]:

$$
\theta_{\gamma} \approx m_{e} c^{2} / E_{e}
$$

with $E_{e}$ being the total energy of the incident electron and $m_{e}$ - its rest mass.

Calculations of the spectral characteristics of bremsstrahlung photons and scattered electrons when a relativistic incident electron beam hits a target have been described earlier [4-6]. Amongst the secondary interactions induced by bremsstrahlung photons with the target material, photonuclear reactions become possible at energies larger than the reaction thresholds. Figure 1 shows the total bremsstrahlung spectra produced by 100 and $200 \mathrm{MeV}$ electron beams incident on a $1.5 \mathrm{~mm}$ thick tungsten target, whereas Fig. 2 shows the bremsstrahlung emissions in an angular range from 0 to $20^{\circ}$ for the case of the $100 \mathrm{MeV}$ electron energy [6]. The resulting energy distribution is used in the Monte-Carlo calculation to generate randomly photons having the proper energy spectrum.

The reaction yield is expressed by the relation:

$$
Y=N_{t} \int_{E_{\mathrm{th}}}^{E_{\max }} \sigma(E) I(E) d E,
$$




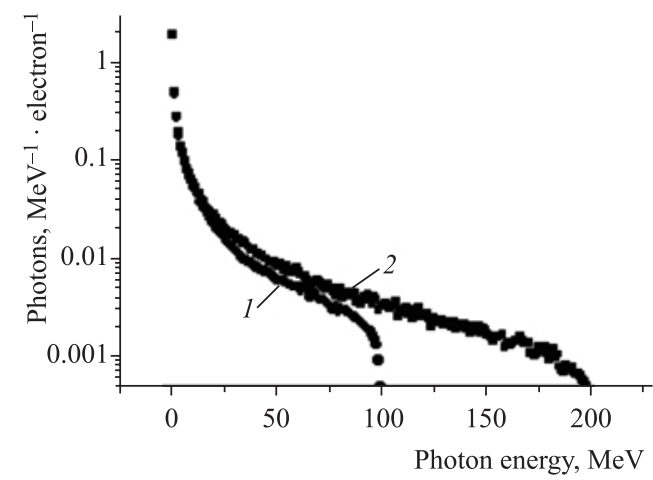

Fig. 1. Simulated bremsstrahlung spectra produced by the 100 (curve 1) and $200 \mathrm{MeV}$ (curve 2) electron beams incident on a $1.5 \mathrm{~mm}$ tungsten target

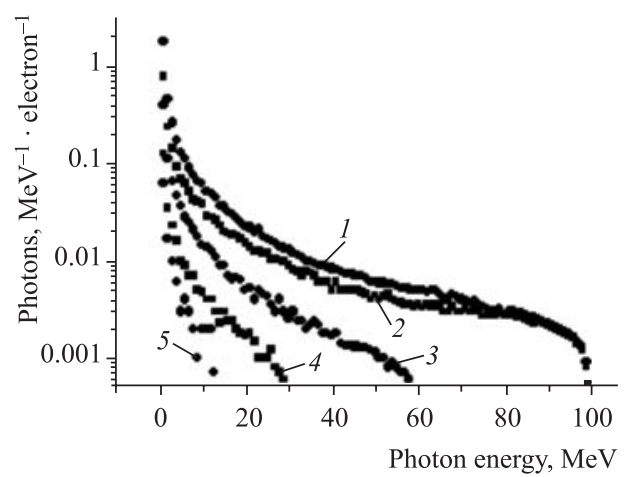

Fig. 2. Bremsstrahlung emission at different angles for the case of using the $100 \mathrm{MeV}$ electron beam: 1 - total spectrum; 2 - from 0 to $5^{\circ} ; 3$ - from 5 to $10^{\circ} ; 4-$ from 10 to $15^{\circ} ; 5$ - from 15 to $20^{\circ}$

where $\sigma(E)$ is the photonuclear reaction cross section, $I(E)$ - the bremsstrahlung-photon intensity and $N_{t}$ - the number of the target nuclei per $\mathrm{cm}^{2}$ :

$$
N_{t}=\zeta\left(N_{A} / A\right) \rho t
$$

Here, $\zeta$ is the isotopic abundance; $N_{A}-$ Avogadro number; $\rho\left(\mathrm{g} / \mathrm{cm}^{3}\right)$ and $t(\mathrm{~cm})$ are the density and thickness of the target, respectively. $E_{\mathrm{th}}$ and $E_{\max }$ are the reaction threshold and the maximal energy of the bremsstrahlung spectrum, i.e., the energy of the accelerated electrons.

For each tungsten isotope the yields of the $(\gamma, n)$ and $(\gamma, 2 n)$ reactions are determined by using the simulated bremsstrahlung spectrum (Fig. 1) and the proper reaction cross sections (Fig. 3).

The photon-neutron yield, $Y_{(\gamma, x n)}$, from the two types of reactions is the sum of the individual yields.

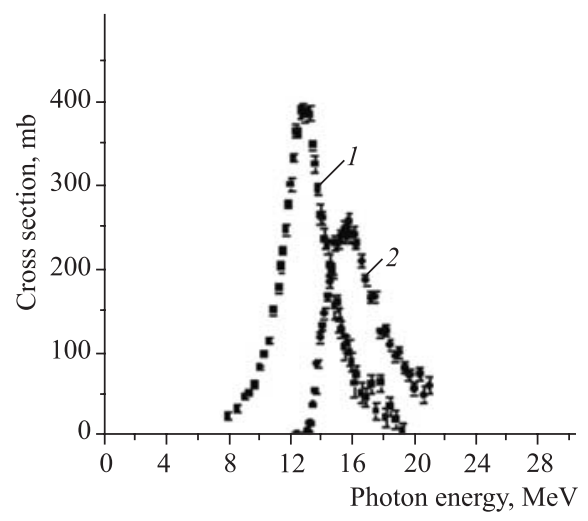

Fig. 3. Excitation functions [2]: $1-{ }^{186} \mathrm{~W}(\gamma, n){ }^{185} \mathrm{~W}$ reaction; $2-{ }^{186} \mathrm{~W}(\gamma, 2 n){ }^{184} \mathrm{~W}$ reaction

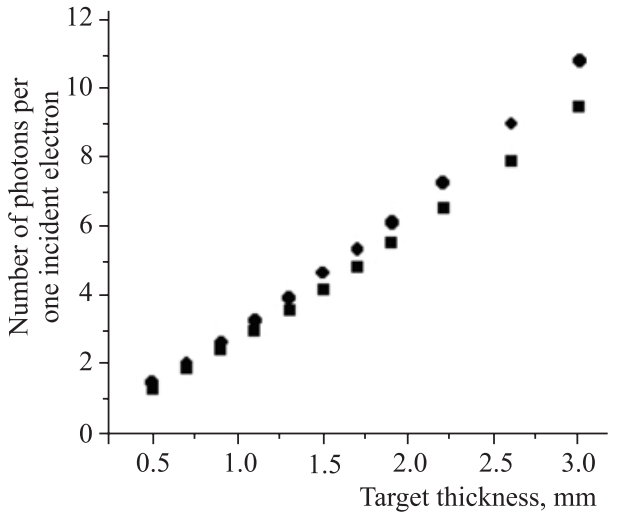

Fig. 4. Production yield of bremsstrahlung photons as a function of the target thickness at $100(\bullet)$ and $200 \mathrm{MeV}$ (•) electron beams 
The total yield is obtained by adding the yields of each isotope properly weighted by their fractional abundance.

The uncertainty of the photon-neutron yield was determined on the basis of the uncertainty of the cross section data and the error in determining the photon intensities from the simulated bremsstrahlung spectrum.

Figure 4 shows the simulation results for the production yield of bremsstrahlung photons, $Y_{(e, x \gamma)}$, called electron-photon yield, as a function of the tungsten target thickness at the $100(\mathbf{})$ and $200 \mathrm{MeV}(\bullet)$ electron-beam energies, i.e., number of the photons produced per one incident electron [6]. This consideration is necessary to determine the electron-neutron yield, which is called neutron yield in this paper, $Y_{(e, x n)}$, directly from information on the electron current used in accelerator operation:

$$
Y_{(e, x n)}=Y_{(e, x \gamma)} \cdot Y_{(\gamma, x n)} \cdot
$$

The obtained results of the individual neutron yields $Y_{(e, x n)}$ at the $1.5 \mathrm{~mm}$ target thickness are summarized in Table. As a result, the total neutron yields of about $(1.01 \pm 0.09) \cdot 10^{-3} \mathrm{n} /$ electron and $(1.17 \pm 0.11) \cdot 10^{-3} \mathrm{n} /$ electron were determined respectively for the cases of using the 100 and $200 \mathrm{MeV}$ bremsstrahlung spectra.

Neutron yields and nuclear data used for yield determination at $1.5 \mathbf{~ m m}$ thickness of tungsten target

\begin{tabular}{|c|c|c|c|c|}
\hline \multirow{2}{*}{$\begin{array}{c}\text { Abundance, } \\
\%\end{array}$} & \multirow{2}{*}{ Reaction } & \multirow{2}{*}{$\begin{array}{c}\text { Threshold } \\
\text { energy, MeV }\end{array}$} & \multicolumn{2}{|c|}{ Yield (n/electron) at } \\
\hline & & & $E_{e}=100 \mathrm{MeV}$ & $E_{e}=200 \mathrm{MeV}$ \\
\hline${ }^{186} \mathrm{~W}(28.60)$ & $\begin{array}{l}{ }^{186} \mathrm{~W}(\gamma, n){ }^{185} \mathrm{~W} \\
{ }^{186} \mathrm{~W}(\gamma, 2 n){ }^{184} \mathrm{~W}\end{array}$ & $\begin{array}{c}7.19 \\
12.95\end{array}$ & $\begin{array}{l}(1.91 \pm 0.13) \cdot 10^{-4} \\
(1.04 \pm 0.09) \cdot 10^{-4}\end{array}$ & $\begin{array}{l}(2.16 \pm 0.13) \cdot 10^{-4} \\
(1.17 \pm 0.09) \cdot 10^{-4}\end{array}$ \\
\hline${ }^{184} \mathrm{~W}(30.70)$ & $\begin{array}{l}{ }^{184} \mathrm{~W}(\gamma, n)^{183} \mathrm{~W} \\
{ }^{184} \mathrm{~W}(\gamma, 2 n){ }^{182} \mathrm{~W}\end{array}$ & $\begin{array}{c}7.41 \\
13.60\end{array}$ & $\begin{array}{l}(2.21 \pm 0.14) \cdot 10^{-4} \\
(1.11 \pm 0.09) \cdot 10^{-4}\end{array}$ & $\begin{array}{l}(2.42 \pm 0.14) \cdot 10^{-4} \\
(1.27 \pm 0.09) \cdot 10^{-4}\end{array}$ \\
\hline${ }^{183} \mathrm{~W}(14.28)$ & $\begin{array}{l}{ }^{183} \mathrm{~W}(\gamma, n){ }^{182} \mathrm{~W} \\
{ }^{183} \mathrm{~W}(\gamma, 2 n){ }^{181} \mathrm{~W}\end{array}$ & $\begin{array}{c}6.19 \\
14.26\end{array}$ & $\begin{array}{l}(0.95 \pm 0.08) \cdot 10^{-4} \\
(0.38 \pm 0.04) \cdot 10^{-4}\end{array}$ & $\begin{array}{l}(1.18 \pm 0.09) \cdot 10^{-4} \\
(0.52 \pm 0.05) \cdot 10^{-4}\end{array}$ \\
\hline${ }^{182} \mathrm{~W}(26.30)$ & $\begin{array}{l}{ }^{182} \mathrm{~W}(\gamma, n)^{181} \mathrm{~W} \\
{ }^{182} \mathrm{~W}(\gamma, 2 n)^{180} \mathrm{~W}\end{array}$ & $\begin{array}{c}8.07 \\
14.75\end{array}$ & $\begin{array}{l}(1.65 \pm 0.12) \cdot 10^{-4} \\
(0.92 \pm 0.07) \cdot 10^{-4}\end{array}$ & $\begin{array}{l}(1.87 \pm 0.12) \cdot 10^{-4} \\
(1.13 \pm 0.08) \cdot 10^{-4}\end{array}$ \\
\hline${ }^{180} \mathrm{~W}(0.12)$ & $\begin{array}{l}{ }^{180} \mathrm{~W}(\gamma, n){ }^{179} \mathrm{~W} \\
{ }^{180} \mathrm{~W}(\gamma, 2 n){ }^{178} \mathrm{~W}\end{array}$ & $\begin{array}{l}8.41 \\
15.35 \\
\text { Total }\end{array}$ & $\begin{array}{l}(0.009 \pm 0.003) \cdot 10^{-4} \\
(0.002 \pm 0.001) \cdot 10^{-4} \\
(1.01 \pm 0.09) \cdot 10^{-3}\end{array}$ & $\begin{array}{l}(0.012 \pm 0.003) \cdot 10^{-4} \\
(0.003 \pm 0.002) \cdot 10^{-4} \\
(1.17 \pm 0.11) \cdot 10^{-3}\end{array}$ \\
\hline
\end{tabular}

In order to easily evaluate the neutron intensity produced as a simple function of the used electron current, the neutron yield should be changed into unit $\left(\mathrm{ns}^{-1} \cdot \mu \mathrm{A}^{-1}\right)$. It should be noted that a current $1 \mu \mathrm{A}$ corresponds to the intensity of about $6.2 \cdot 10^{12} \mathrm{~s}^{-1}$. Therefore, two yield values obtained above are, respectively, equivalent to $(6.26 \pm 0.56) \cdot 10^{9}$ and $(7.25 \pm 0.68) \cdot 10^{9} \mathrm{~ns}^{-1} \cdot \mu \mathrm{A}^{-1}$. For example, for the case of using the $100 \mathrm{MeV}$ electron beam at a typical current $100 \mu \mathrm{A}$ we can estimate the two following results for the secondary neutron emission:

i. A total neutron intensity of about $(6.26 \pm 0.56) \cdot 10^{11} \mathrm{~s}^{-1}$ is able to be produced during the accelerator operation. 


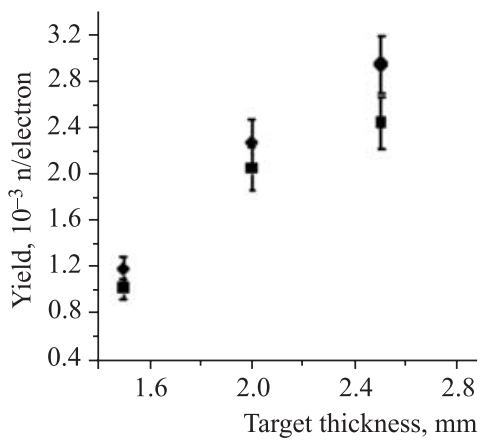

Fig. 5. Neutron yields as a function of target thickness at $100(\mathbf{-})$ and $200 \mathrm{MeV}(\bullet)$ electron energies

ii. If it is supposed that the neutron measurement is performed at a distance $10 \mathrm{~m}$ from the target by using a detector with $30 \mathrm{~cm}$ radius. The solid angle covered by this detector is about $0.25 \mathrm{mrad}$. Therefore, the neutron amount which is able to reach the detector is evaluated as

$$
\begin{aligned}
N=(6.26 \pm 0.56) \cdot 10^{11} \cdot & 0.25 \cdot 10^{-3} / 4 \pi= \\
= & (1.25 \pm 0.11) \cdot 10^{7} \mathrm{~s}^{-1} .
\end{aligned}
$$

To consider dependence of the neutron yield on the target thickness, we have determined the yield at two other values: 2.0 and $2.5 \mathrm{~mm}$ of thickness for both cases of the 100 and $200 \mathrm{MeV}$ electron energies. The results presented in Fig. 5 show a gradual increase of the yield with the increased target thickness. However, it can be seen that increasing rate is faster in the region from 1.5 to $2.0 \mathrm{~mm}$ compared with that from 2.0 to $2.5 \mathrm{~mm}$, especially for the case of the $100 \mathrm{MeV}$ electron energy. This is because our calculations have shown that the photon-neutron yield, $Y_{(\gamma, x n)}$, is maximum at a critical value which is more or less equal to $2.0-2.2 \mathrm{~mm}$, while the electron-photon yield, $Y_{(e, x \gamma)}$, is still a gradually increasing function of the thickness in the considered range as shown in Fig. 4.

\section{NEUTRON ENERGY AND ANGULAR DISTRIBUTIONS}

Besides evaluating the total neutron yield, the Monte-Carlo method makes it possible to calculate the energy and angular distributions of the produced neutrons once the angular dependence of the photonuclear reaction cross section is known. In case of the $(\gamma, n)$ reaction,

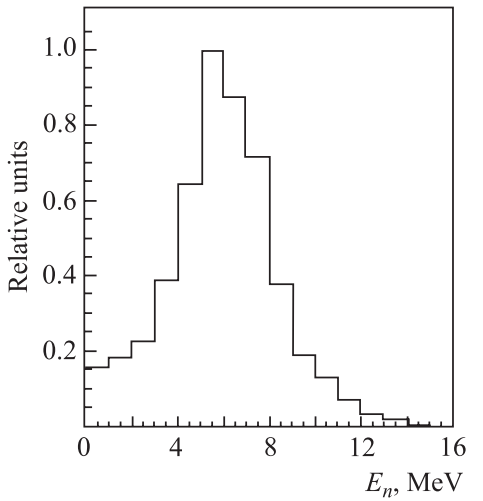

Fig. 6. The energy spectrum of neutrons emitted by the ${ }^{186} \mathrm{~W}(\gamma$, $n)^{185} \mathrm{~W}$ reaction the energy-momentum conservation relates the neutron energy $E_{n}$ to its production angle $\theta_{n}$ via

$$
\begin{aligned}
M_{r}^{2}= & \left(\Delta E+M_{n}\right)^{2}-2 \Delta E\left(E_{n}+M_{n}\right)+ \\
& +2 E_{\gamma}\left(\Delta E-E_{n}-\cos \theta_{n} \sqrt{E_{n}^{2}-M_{n}^{2}}\right),
\end{aligned}
$$

where $M_{r}$ is the mass of the final state nucleus $(c=1)$, $\Delta E=\left(M_{t}-M_{r}-M_{n}\right) c^{2}$ with $M_{t}$ being the mass of the target nucleus, $M_{n}$ - that of the neutron and $E_{\gamma}$ the incident photon energy. Note that the neutron kinetic energy is $T_{n}=E_{n}-M_{n}$.

The angular distribution of the photonuclear cross section is taken from $[9,10]$. It has a form $P\left(\theta_{n}\right)=$ $A+B \cdot \sin ^{2} \theta_{n}$ with $B / A=2.0 \pm 0.5[10]$. We justify this choice by remarking that the photons which are active in producing neutrons have energies concentrated above threshold whatever the electron energy. 
The bremsstrahlung radiation is taken to be exactly forward. The angular distribution of the produced neutrons is therefore the same as $[9,10]$, while the neutron energy spectrum is displayed in Fig. 6.

\section{CONCLUSION}

We have used Monte-Carlo calculations to evaluate the total neutron yields from photonuclear reactions $(\gamma, n)$ and $(\gamma, 2 n)$ induced by bremsstrahlung photons radiated by the 100 and $200 \mathrm{MeV}$ electron beams incident on the tungsten target. The consideration has been performed at three values 1.5, 2.0 and $2.5 \mathrm{~mm}$ of the target thickness, where the bremsstrahlung spectra were calculated and folded with the cross sections of the $(\gamma, n)$ and $(\gamma, 2 n)$ reactions for the various tungsten isotopes present in the target. The energy and angular distributions of the produced neutrons were calculated under the assumptions that the bremsstrahlung radiation is exactly forward and the direct interaction model can be used to consider the neutron emission.

The neutron yield is given as a product of the electron-photon yield, $Y_{(e, x \gamma)}$ and the photon-neutron yield, $Y_{(\gamma, x n)}$. In this work we determined the produced neutron yields at three above-mentioned values of the target thickness. This enabled us to study the dependence of the neutron yield on the thickness, in which we found a critical thickness of more less 2.0-2.2 $\mathrm{mm}$ for the photon-neutron yield, while the electron-photon yield is still shown as a gradually increasing function of the thickness. More essentially, the obtained results can be easily used to evaluate the neutron emission as a secondary source produced when accelerator is operated at a given electron current.

In reality, there may be additional contributions to the neutron production from other types of nuclear reactions induced by the bremsstrahlung photons such as $(\gamma, n p)$ and $(\gamma, x n)$ reactions with high neutron multiplicity as well as spallation processes. However, their contribution to the total neutron yield should not exceed a few percents as they imply higher energy incident photons associated with lower bremsstrahlung photon intensities.

Acknowledgements. Vietnamese authors would like to express sincere thanks for the financial support by the Vietnam National Foundation for Science and Technology Development.

\section{REFERENCES}

1. Kapisa C.P., Melekhin V.N. Microtron. M.: Nauka, 1969.

2. IAEA Photonuclear Data Library. http://www-nds.iaea.org/photonuclear/ http://cdfe.sinp.msu.ru/exfor/index.php

3. Belushkin A. V. Report on Scientific Programme of the Frank Laboratory of Neutron Physics. Dubna, 2006.

4. Khai N. T., Thiep T.D. // Commun. in Phys. 2003. V.13, No.3. P. 149.

5. Khai N. T. et al. // Part. Nucl., Lett. 2008. V.5, No. 5(147). P. 736.

6. GEANT4. http://www.slac.stanford.edu/comp/physics/geant4/geant4.html 
40 Khai N. T. et al.

7. Marmier P., Sheldon E. Physics of Nuclei and Particles. V.1. N. Y.; London: Acad. Press, 1969.

8. Leo W. R. Techniques for Nuclear and Particle Physics Experiments. Berlin; Heidelberg: SpringerVerlag, 1987; 1994.

9. Tagliabue F., Goldemberg J. // Nucl. Phys. 1961. V.23. P. 144.

10. Price G. E. et al. // Phys. Rev. 1954. V.93. P. 1279.

Received on March 23, 2009. 